Meta

Journal des traducteurs

Translators' Journal

\title{
Arrêté du 18 février 1987 relatif à l'enrichissement du vocabulaire économique et financier
}

\section{Édouard Baladur}

Volume 34, numéro 2, juin 1989

URI : https://id.erudit.org/iderudit/001952ar

DOI : https://doi.org/10.7202/001952ar

Aller au sommaire du numéro

Éditeur(s)

Les Presses de l'Université de Montréal

ISSN

0026-0452 (imprimé)

1492-1421 (numérique)

Découvrir la revue

Citer cet article

Baladur, É. (1989). Arrêté du 18 février 1987 relatif à l'enrichissement du vocabulaire économique et financier. Meta, 34(2), 284-301.

https://doi.org/10.7202/001952ar d'utilisation que vous pouvez consulter en ligne. 


\section{ARRÊTÉ DU 18 FÉVRIER 1987 RELATIF À L'ENRICHISSEMENT DU VOCABULAIRE ÉCONOMIQUE ET FINANCIER}

Le ministre d'État, ministre de l'Économie, des Finances et de la Privatisation, et le ministre de l'Éducation nationale, française;

Vu la loi $\mathrm{n}^{\circ}$ 75-1349 du 31 décembre 1975 relative à l'emploi de la langue

Vu le décret $n^{\circ}$ 84-91 du 9 février 1984 instituant un commissariat général de la langue française; française ;

Vu le décret $n^{\circ} 86-439$ du 11 mars 1986 relatif à l'enrichissement de la langue

Vu l'arrêté du 29 novembre 1973 relatif à la terminologie économique et financière;

Vu l'arrêté du 29 novembre 1985 portant création d'une nouvelle commission de terminologie du ministère de l'Économie, des Finances et du Budget;

Sur proposition de la commission de terminologie du ministère de l'Économie, des Finances et de la Privatisation;

Après avis favorable du Commissariat général de la langue française et du Conseil international de la langue française,

\section{Arrêtent :}

Art. $1^{\mathrm{er}}$. - Les expressions et termes inscrits en annexe I du présent arrêté sont approuvés.

Ils s'ajoutent aux expressions et termes inscrits sur la première liste de terminologie économique et financière annexée à l'arrêté du 29 novembre 1973.

Ils devront être obligatoirement utilisés;

Dès la publication du présent arrêté :

- dans les décrets;

- dans les arrêtés, circulaires, instructions et directives des ministres;

- dans les correspondances et documents de quelque nature que ce soit, qui émanent des administrations, services ou établissements publics de l'État, dans les informations et présentations de programmes de radiodiffusion ou de télévision;

- dans les textes des marchés et contrats auxquels l'État ou les établissements publics de l'État sont parties; 
- dans les ouvrages d'enseignement, de formation ou de recherche utilisés dans les établissements, institutions ou organismes dépendant de l'État, placés sous son autorité ou soumis à son contrôle ou bénéficiant de son concours financier à quelque titre que ce soit;

Dans un délai de six mois après la publication de cet arrêté, dans les textes, documents et inscriptions mentionnés dans la loi no 75-1349 du 31 décembre 1975 relative à l'emploi de la langue française.

Art. 2. - Les expressions et termes inscrits en annexe II du présent arrêté sont recommandés.

Art. 3. - Il est joint au présent arrêté une annexe III constituée d'un index alphabétique des termes étrangers remplacés.

Art. 4. - Le présent arrêté assorti de ses annexes sera publié au Journal officiel de la République française.

Fait à Paris, le 18 février 1987.

ÉDOUARD BALLADUR Ministre d'État, ministre de l'Économie, des Finances et de la Privatisation

RENÉ MONORY

Ministre de l'Éducation nationale

\section{Annexe I}

Liste des termes d'usage obligatoire

B.A.B.

Voir: $\quad$ Bord à bord

banque de détail, n.f.

Domaine: Finances/Banque.

Définition: Ensemble des opérations bancaires de faible montant unitaire faites essentiellement avec les particuliers, les professions libérales et les petites entreprises.

Anglais: retail banking.

billet à ordre, n.m.

Domaine: Finances.

Définition: Écrit par lequel un débiteur s'engage à payer, à une date déterminée, une certaine somme au bénéficiaire ou à son ordre.

Note : Le billet à ordre est négociable.

Anglais: promissory note. 
billet de trésorerie, $\mathbf{n} . \mathbf{m}$.

Domaine: Finances / Banque et monnaie.

Définition: Billet au porteur négociable émis par une entreprise autre qu'un établissement de crédit pour se procurer des capitaux à court terme.

Notes: 1. Un établissement de crédit émet des certificats de dépôt (certificates of deposit ou $C D^{\prime} S$ ) dont la définition est identique.

: 2. Le terme billet de trésorerie ne doit pas être traduit par treasury bill $(T B)$.

Anglais: $\quad$ commercial paper.

bord (à), loc.

Domaine: Commercial/Commerce international.

Définition: Se dit d'une marchandise prise en charge à bord du navire au port de déchargement.

Note: $\quad$ Le reste de la phrase précise le lieu de la prise en charge. Exemple : à bord

Marseille.

Anglais: ex ship.

bord à bord, loc.

Abréviation: B.A.B., loc.

Domaine: Commerce/Commerce extérieur.

Définition: Se dit du prix entendu pour une marchandise mise à bord du navire et également reprise à bord, aux frais et aux risques des chargeurs ou des réceptionnaires.

Note: Une cotation donnée pour une ligne maritime sur la base B.A.B. indique

Anglais: $\quad$ que toutes les mand out (F.I.O.)

brillant universel, n.m.

Abréviation: B.U.

Domaine: Finances / Monnaie.

Définition: Monnaie de collection frappée dans les mêmes conditions que les pièces de monnaie courantes mais avec des matrices neuves.

Note: $\quad$ N'ayant pas circulé, elles conservent le brillant d'une pièce neuve.

Anglais : brilliant uncirculated.

B.U.

Voir: $\quad$ Brillant universel.

capitaux flottants, n.m.pl.

Domaine: Finances/Banques.

Définition : Capitaux en quête de placements permettant la meilleure rentabilité à court terme quels que soient la place financière, la monnaie et le placement.

Anglais: $\quad$ hot money.

CATIF

Voir: Contrat à terme d'instrument financier.

C.C.R.

Voir: $\quad$ Coefficient de capitalisation des résultats. 


\section{Centre commercial, n.m.}

Domaine: Commerce/Réseau de distribution commerciale.

Définition: Grande surface de vente rassemblant plusieurs commerces et comprenant un parc de stationnement.

Anglais: Shopping-center.

C. et $\mathbf{F}$.

Voir: $\quad$ Coût et frêt.

chambre de compensation, n.f.

Domaine: Finances/Banque.

Définition: Organisme chargé d'assurer la compensation des soldes créditeurs entre banques.

Notes: 1. Dans la plupart des pays, y compris la France, seuls y participent directement les principaux établissements, les autres établissements s'y faisant représenter par l'un deux.

2. Pour les marchés à terme d'instruments financiers et de marchandises, les organismes de compensation sont chargés d'assurer la correspondance entre les positions débitrices et créditrices des différents intervenants, de compenser les soldes et de s'assurer du versement des appels de dépôts de garantie et de marges.

Anglais: $\quad$ clearing house.

clause rouge, n.f.

Domaine: Finances / Banque.

Définition: En matière de crédit documentaire, clause particulière incluse à la demande du donneur d'ordre dans un crédit, et autorisant la banque notificatrice ou confirmatrice à effectuer des avances au bénéficiaire avant présentation des documents ou sur présentation de documents provisoires.

Anglais: red clause.

coefficient de capitalisation des résultats, n.m.

Abréviation: C.C.R., n.m.

Domaine: Finances/Bourse.

Définition: Coefficient par lequel il convient de multiplier le bénéfice net par action pour retrouver le cours coté.

Anglais: price earning ratio.

commission d'engagement, n.f.

Domaine: Finances/Banque.

Définition: Commission perçue par un syndicat bancaire rémunérant la partie non utilisée d'un crédit que le syndicat s'est engagé à mettre à la disposition d'un emprunteur.

Anglais: commitment fee.

commission de chef de file, $n . f$.

Domaine: Finances/Banque.

Définition: Commission perçue à l'occasion d'une opération dont on a assuré la direction.

Anglais: management fee. 
commission de garantie, n.f.

Domaine: Finances/Banque.

Définition: Commission payée par un emprunteur à un syndicat d'établissements de crédit et/ou d'institutions financières en rémunération de la garantie de bonne fin de l'opération.

Anglais: $\quad$ underwriting fee.

commission de gestion, n.f.

Domaine: Finances/Banque.

Définition: Commission perçue en rémunération de la gestion de titres ou de capitaux confiée par un tiers.

Anglais: agency fee.

commission de placement, n.f.

Domaine: Finances/Banque.

Définition: Commission payée par un émetteur à un syndicat d'établissements de crédit et/ou d'institutions financières en rémunération de la diffusion et du placement de ses titres.

Anglais: underwriting fee.

commission immédiate, n.f.

Domaine: Finances/Banque.

Définition: Commission payée en totalité à la signature du contrat de crédit ou d'emprunt par l'emprunteur aux établissements de crédit participant au tour de table.

Anglais: flatfee.

comité de restructuration, n.m.

Domaine: Finances/Banque.

Définition: Dans le cadre particulier de la restructuration de la dette de certains pays, comité réunissant les banques chefs de file des crédits à ces pays pour organiser, administrer le financement additionnel et négocier la procédure de rééchelonnement de la dette.

Anglais: $\quad$ steering committee, advisory committee.

comptant, adj.

Domaine: Finances/Banque.

Définition: Paiement immédiat quel que soit le moyen de paiement utilisé dès lors que Anglais : cash.

contrat à terme d'instrument financier, n.m.

Abréviation: CATIF, n.m.

Domaine: Finances.

Définition: Engagement ferme moyennant dépôt de garantie d'acheter ou de vendre à une date fixée, à un prix convenu, une certaine quantité d'un instrument financier. 
coût et fret, loc.

Abréviation: C. et F. loc.

Domaine: Commerce/Commerce extérieur.

Définition: Se dit du prix entendu pour une marchandise arrivée au port de destination, le fret étant payé mais l'assurance n'étant pas couverte.

Note: Utilisée dans la cotation des prix, cette locution est suivie du nom du port de destination convenu.

Anglais: cost and freight $(C F)$.

crédit ponctuel, n.m.

Domaine: Finances/Banque.

Définition: Crédit à court terme dont le montant et les conditions sont négociés au coup par coup.

Note: Le taux est déterminé en fonction du marché du moment.

Anglais: spot credit.

crédit à taux révisable, n.m.

Domaine: Finances/Banque.

Définition: Crédit généralement à moyen terme pour lequel les échéances d'intérêt sont effectuées à taux renouvelable à des intervalles déterminés (en général trois ou six mois).

Anglais: $\quad$ rollover credit (ne pas confondre avec revolving).

crédit de restructuration, n.m.

Domaine: Finances/Banque.

Définition: Dans le cadre particulier de la restructuration de la dette de certains pays, financement additionnel à moyen terme réparti entre les banques au prorata de leurs encours sur le pays considéré et associé à un rééchelonnement.

Note: Le crédit de restructuration se fait dans le cadre d'une procédure administrée par les banques elles-mêmes.

Anglais: $\quad$ new-money (ne pas confondre avec fresh-money).

déchéance du terme, n.f.

Domaine: Finances/Banque.

Définition: Exigence anticipée d'une créance motivée, par exemple, par le nonrespect, par le débiteur, de ses obligations contractuelles.

Anglais: event of default.

défaut croisé, n.m.

Domaine: Finances / Banque.

Définition: Clause établissant une relation entre un crédit et toute autre obligation de l'emprunteur concernant d'autres crédits ou emprunts, de telle manière qu'une défaillance de sa part, dans l'une quelconque de ses obligations, entraîne la déchéance du terme, et donc l'exigibilité anticipée dudit crédit.

Anglais: $\quad$ cross default.

démembrement, n.m.

Domaine: Finances/Bourse.

Définition: Technique consistant à scinder un titre en plusieurs titres. 
Notes: 1. Par exemple :

- une action peut être scindée en un certificat d'investissement et un certificat de droit de vote;

- une obligation peut être scindée en autant de titres qu'il y a d'échéances d'intérêt et de remboursement de capital.

2. Chaque titre ainsi créé par démembrement peut être cédé et coté séparément. Actuellement dans la pratique, en ce qui concerne les actions, seuls sont cédés et cotés séparément les certificats d'investissement.

Anglais: stripping.

départ-usine, loc.

Domaine: Commerce/Commerce extérieur.

Définition: Se dit d'une marchandise prise en charge à la sortie de l'usine.

Anglais: exworks.

\section{dépôt de garantie, n.m.}

Domaine: Finances/Bourse.

Définition: Fraction du prix d'une marchandise, d'un service ou d'un actif financier achetés à terme, versée au moment de la conclusion du contrat.

Note: L'achat à terme dans une bourse de commerce donne lieu au dépôt, à titre de garantie, auprès de la chambre de compensation, d'une somme comprise le plus souvent entre 5 et $25 \%$ de la valeur de la marchandise. En France, ce dépôt est désigné par le terme anglo-saxon deposit, alors

Anglais: deposit.

D.E.P.S.

Voir:

Dernier entré, premier sorti.

dernier entré, premier sorti, n.m.

Abréviation: D.E.P.S., n.m.

Domaine: Comptabilité/Gestion des stocks.

Définition: Système dans lequel la comptabilisation d'un élément de stock se fait sur Anglais: $\quad$ last in, first out (L.I.F.O.).

division, n.f.

Domaine:

Finances/Bourse.

Définition : Fractionnement d'une action par division de son nominal.

Note: Cette procédure a pour conséquence également le fractionnement de son cours de bourse dans les mêmes proportions. Elle permet ainsi une diffusion plus large du titre.

Anglais: splitting.

écart d'inflation, n.m.

Domaine: Économie/Finances.

Définition: Différence entre le taux de hausse des prix d'un pays et celui d'un de ses partenaires économiques, ou de l'ensemble, ou d'un sous-ensemble de ceux-ci. 
Note: Le terme «différentiel d'inflation» parfois employé doit être écarté au bénéfice d' «écart d'inflation».

épreuve, n.f.

Domaine: Finances/Monnaies.

Définition: Monnaie de collection frappée sur un flan qui a subi un polissage poussé au moyen de matrices dont les parties les plus hautes sont polies; les

Note : reliefs de la pièce sont rendus mais par le sablage des creux de la matrice.

Anglais : Ces pièces sont soumises unité par unité à un contrôle visuel.

espèces, n.f.pl.

Domaine: Finances / Banques.

Définition: Moyen de paiement ayant un pouvoir libératoire illimité entraînant l'extinction immédiate de la dette.

Anglais: cash.

étalon-or lingot, n.m.

Domaine: Finances / Marché de l'or.

Définition: Système monétaire international à convertibilité interne limitée dans lequel les banques centrales assurent l'échange de la monnaie nationale contre de l'or sous forme de lingots ou de barres.

Note : $\quad$ Ce système est issu des accords de Gênes de 1922.

Anglais : gold bullion standard.

étalon de change-or, n.m.

Domaine: Finances/Marché de l'or.

Définition: Système monétaire international dans lequel les banques centrales conservent concurremment, dans les réserves publiques de change, de l'or et des devises convertibles en or, mais sans assurer la convertibilité interne.

Anglais: $\quad$ gold exchange standard.

expomarché, n.m.

Domaine: Commerce/Commerce extérieur.

Définition: Bâtiment d'exposition permanente destiné aux acheteurs professionnels où des fabricants, importateurs, grossistes et distributeurs de certains produits peuvent louer pour plusieurs années une aire de présentation.

Anglais: $\quad$ trade mart.

extraterritorial, adj.

Domaine: Finances/Banque.

Définition: Qualifie les activités bancaires et financières domicilićes dans des places étrangères.

Anglais: off-shore.

F.A.B.

Voir: $\quad$ Franco à bord. 
fioul, n.m.

Domaine: Pétrole.

Définition: Huile lourde ou moyenne tirée de la distillation du pétrole. Exemples:

fioul lourd, fioul domestique.

Anglais: fuel-oil.

fixage, n.m.

Domaine: Finances/Banque.

Définition: Procédure de cotation par laquelle est fixé le cours d'une valeur telle que valeur mobilière, or ou devise.

Notes: 1. Cette procédure consiste à fixer périodiquement le cours d'une valeur à partir de la confrontation, à un moment donné, des offres et des demandes de telle manière que le maximum d'offres et de demandes puisse être satisfait à ce cours. D'autres procédures de cotation sont: la cotation continue; la cotation par prix offert, prix demandé.

2. Par extension, ce terme recouvre parfois également le cours lui-même résultant de la procédure. Dans ce sens, il est préférable d'employer le terme «cours».

Anglais: fixing.

marché à terme, n.m.

Domaine: Marchés de capitaux et de marchandises.

Définition: Marché sur lequel des transactions donnent lieu à paiement et livraison des actifs financiers ou des marchandises à une échéance ultérieure.

Note: $\quad$ Sur la bourse des valeurs en France, le marché s'appelle «Règlement mensuels (RM).

Anglais : $\quad$ forward market.

marché à terme d'instruments financiers, n.m.

Abréviation: MATIF, n.m.

Domaine: Finances / Bourse.

Définition: Marché à terme sur lequel se négocient uniquement les instruments financiers représentatifs d'actifs financiers ou monétaires par multiple de contrats normalisés.

Anglais: financial futures market.

marché au comptant, n.m.

Domaine: $\quad$ Marchés de capitaux et de marchandises.

Définition: Marché sur lequel les transactions donnent lieu à paiement et livraison non différés d'actifs financiers ou de marchandises.

Note: $\quad$ S'agissant de marchandises, ces marchés portant généralement sur des quantités marginales, les opérateurs n'y interviennent que de manière ponctuelle.

Anglais: $\quad$ spot-market.

marché baissier, n.m.

Domaine: Finances / Bourse.

Définition: Marché dont la tendance est foncièrement orientée à la baisse.

Anglais: bearish market. 
marché de contrats à terme, i.m.

Domaine: Finances / Bourse.

Définition: Marché à terme où s'échangent des contrats normalisés portant sur des instruments financiers ou des marchandises, utilisés à des fins de couverture de positions au comptant, d'arbitrage ou de spéculation.

Anglais: futures market.

\section{marché haussier, n.m.}

Domaine: Finances / Bourse.

Définition: Marché dont la tendance est foncièrement orientée à la hausse.

Anglais: bullish market.

marchéage, n.m.

Domaine: Commerce/Commercialisation.

Définition: Dosage et cohérence de l'ensemble des actions commerciales.

Anglais: $\quad$ marketing mix.

marge, n.f.

Domaine: Finances / Banque.

Définition: Marge calculée en pourcentage s'ajoutant soit à un taux de référence, par exemple le taux interbancaire offert (T.I.O), ou à un prix d'achat et couvrant notamment la rémunération et le risque de la banque prêteuse.

Anglais: $\quad$ spread.

MATIF

Voir :

Marché à terme d'instruments financiers.

mercaticien, n.m.

mercaticienne, n.f.

Domaine: Commerce/Commercialisation.

Définition: Spécialiste de la mercatique.

mercatique, n.f.

Domaine: Commerce/Commercialisation.

Définition: Ensemble des actions destinées à détecter les besoins et à adapter en conséquence et de façon continue la production et la commercialisation.

Note: De façon plus développée, la mercatique est conçue comme l'ensemble des actions qui ont pour objectif de prévoir ou de constater - les besoins du consommateur en telle catégorie de produits ou de services, et de réaliser l'adaptation continue de l'appareil productif et de l'appareil commercial d'une entreprise aux besoins ainsi déterminés.

Anglais: marketing.

multidevise, adj.

Domaine: Finances / Banque.

Définition: Se dit d'une opération ou d'un contrat dont les clauses financières peuvent mettre en jeu plusieurs devises.

Anglais: multicurrency. 
négociations Kennedy, n.f.pl.

Définition: Ensemble des négociations commerciales multilatérales qui se sont déroulées dans le cadre de l'Accord général sur les tarifs et le commerce (AGETAC) à la suite du vote, en octobre 1961, sur l'initiative du Président Kennedy, du Trade Expansion Act par le Congrès des États-Unis d'Amérique.

Anglais: Kennedy round.

\section{notation, n.f.}

Domaine: Finances.

Définition: Évaluation qualitative d'une société ou d'un titre émis par une société, notamment au regard de sa solvabilité, mais ne constituant jamais une recommandation d'achat ou de vente.

Note : La notation est opérée par des organismes spécialisés.

Anglais: rating.

option sur contrats à terme, n.f.

Domaine: Finances/Bourse.

Définition: Contrat qui confère, contre paiement immédiat d'une prime, la faculté d'acheter ou de vendre, pendant une période limitée, à un prix défini à l'avance, une certaine quantité d'instruments financiers ou de marchan-

Anglais: futures option.

option d'achat, n.f.

Domaine: Finances/Bourse.

Définition: Contrat qui confère, contre paiement immédiat d'une prime, la faculté, mais non l'obligation d'acheter pendant une période limitée, à un prix défini à l'avance, une certaine quantité de titres cotés sur le marché de

Anglais : call option.

option de vente, n.f.

Domaine: Finances / Bourse.

Définition: Contrat qui confère, contre paiement immédiat d'une prime, la faculté, mais non l'obligation de vendre, pendant une période limitée, à un prix défini à l'avance, une certaine quantité de titres cotés sur le marché à

Anglais: put option.

ordre lié, n.m.

Domaine: Finances/Bourse.

Définition: Opération d'achat et de vente d'un même donneur d'ordre portant:

- soit sur le même titre pour un montant donné sur deux échéances différentes;

- soit sur des titres différents pour un même montant sur la même

Anglais: straddle. 
paiement différentiel, n.m.

Domaine: Économie politique et sociale.

Définition: Aide au revenu des producteurs agricoles, consistant à verser à chaque producteur et pour chaque produit une indemnité égale à la différence entre le prix perçu pour un produit donné sur le marché libre et un prix garanti qui est fixé annuellement et considéré comme rémunérateur pour les producteurs.

Anglais: deficiency payment.

payer-prendre, n.m.

Domaine: Commerce/Réseau de distribution commerciale.

Définition: Technique ou vente en libre service appliquée à la vente en gros.

Note: Cette technique de vente vise à une compression des frais généraux par une stricte limitation des services rendus: les acheteurs viennent euxmêmes enlever la marchandise à l'entrepôt du vendeur, paient comptant et se chargent de l'emballage et de l'acheminement.

Anglais : cash and carry.

P.E.P.S.

Voir: $\quad$ Premier entré, premier sorti.

place extraterritoriale, $n . f$.

Domaine: Finances/Banque.

Définition: Place sur laquelle des agents économiques, notamment des banques, bénéficient de conditions d'exercice particulières, principalement sur le plan fiscal et du contrôle des changes, de sorte qu'ils y domicilient certaines opérations internationales traitées ailleurs et avec des non-résidents.

Anglais: $\quad$ off-shore place.

premier entré, premier sorti, n.m.

Abréviation: P.E.P.S., n.m.

Domaine: Comptabilité/Gestion des stocks.

Définition: Système dans lequel la comptabilisation d'un élément de stock consommé se fait sur la base du prix du premier élément identique entré en stock.

Anglais: first in, first out (F.I.F.O.).

(à) quai, dédouané, loc.

Domaine: Commerce/Commerce extérieur.

Définition: Se dit pour préciser, dans un contrat de vente ou de transport, que toute taxe, charge ou droit devant être acquitté lors et du fait de l'importation des marchandises dont il est question, est imputable au vendeur.

Note: $\quad$ Cette expression peut être suivie du nom du port.

Anglais: $\quad$ ex quay ... duty paid.

(à) quai, non dédouané, loc.

Domaine: Commerce/Commerce extérieur.

Définition: Se dit pour préciser, dans un contrat de vente ou de transport, que toute taxe, charge ou droit devant être acquitté lors et du fait de l'importation des marchandises dont il est question, est imputable à l'acheteur à qui la responsabilité est ipso facto transférée. 
Note: $\quad$ Il est habituel de compléter cette expression par la désignation du port d'entrée: si le port convenu est Marseille, l'expression devient: «à quai, non dédouané Marseille».

Anglais: ex quay ... duties on buyers account.

redevance, n.f.

Domaine: Commerce/Commerce extérieur.

Définition: Somme versée à échéances périodiques en contrepartie d'un avantage Anglais: royalty.

rembours, n.m.

Domaine: Commerce/Commerce extérieur.

Définition: Régime douanier qui permet, pour des marchandises étrangères réexportées soit en l'état, soit après transformation ou incorporation dans un autre produit, d'accorder le remboursement total ou partiel des droits de douane

Anglais: $\quad d r a w b a c k$

remboursement, in fine, n.m.

Domaine: Finances/Banque.

Définition: Remboursement du principal d'un emprunt ou d'un crédit en un seul versement à son échéance.

Anglais: $\quad$ bullet (ne pas confondre avec balloon).

répartition, n.f.

Domaine: Général.

Synonyme: Ventilation, n.f.

Définition: Opération portant sur des marchandises ou sur du courrier et consistant à

diriger chaque colis ou chaque pli vers son destinataire.

dispatching

report en arrière de déficit, n.m.

Domaine: Finances.

Définition: Possibilité offerte, sous certaines conditions, aux entreprises soumises à l'impôt sur les sociétés de reporter un déficit subi pendant un exercice sur les bénéfices non distribués d'exercices précédents en faisant apparaître un excédent d'impôt sur les sociétés et donc une créance d'égal montant sur le Trésor.

Anglais : loss carry back.

savoir-faire, n.m.

Domaine: Gestion/Gestion de la production.

Définition: Ensemble des connaissances techniques nécessaires pour la bonne utilisation d'un dispositif ou d'une machine.

Anglais: know-how. 
supérette, n.f.

Domaine: Commerce / Réseau de distribution commerciale.

Définition: Magasin en libre-service de $120 \mathrm{~m}^{2}$ à $400 \mathrm{~m}^{2}$, consacré surtout à l'alimentation.

Note: $\quad$ Son rayonnement est limité au quartier et sa clientèle le fréquente en général de manière quotidienne.

Anglais : $\quad$ small supermarket.

syndicat de prise ferme, n.m.

Domaine: Finances/Banque.

Définition: Ensemble de banques et/ou de sociétés financières réunies pour participer à une émission obligatoire (emprunt syndiqué), quels que soient l'émetteur, la monnaie et la place, en s'engageant à souscrire une part déterminée de l'emprunt, ce qui garantit l'émetteur de recevoir le produit de son émission, même en cas de placement difficile dans le public.

Note: Un tel syndicat peut être aussi constitué à l'occasion d'une augmentation de capital, notamment préplacée. Le terme employé est plutôt «syndicat de garantie».

Voir aussi : tour de table.

Anglais: pool, underwriting group.

taux interbancaire offert, n.m.

Abréviation: T.I.O., n.m.

Domaine: Finances/Banque.

Définition: Taux d'intérêt que les banques offrent pour leurs prêts à des banques de premier rang, sur une place donnée, dans une monnaie et pour une échéance définies.

Note: $\quad$ Il peut y avoir autant de T.I.O. que de banques.

Anglais: $\quad I B O R$ (interbank offered rate). La lettre placée en préfixe permet de connaître la place d'origine, par exemple $L I B O R$ pour Londres, PIBOR pour Paris.

T.I.O.

Voir: $\quad$ Taux interbancaire offert.

tour de table, n.m.

Domaine: Finances / Banque.

Définition: Réunion de différents apporteurs de capitaux, sans appel public à l'épargne, ayant pour objet la répartition des capitaux apportés.

Notes: 1. En matière de crédit (en monnaie nationale ou en devise), le tour de table est constitué exclusivement par des banques et/ou des sociétés financières.

2. Pour le capital social (constitution, augmentation) d'un organisme, quel qu'il soit, le tour de table peut comprendre des personnes physiques et morales. Lorsque le capital social d'un organisme est détenu intégralement par un même actionnaire, il n'y a pas lieu d'employer l'expression tour de table.

Voir aussi: $\quad$ syndicat de prise ferme.

Anglais: pool. 
transport maritime à la demande, n.m.

Domaine: Transport/Transport maritime.

Définitions: 1. Mode d'exploitation d'un navire consistant à l'utiliser sans itinéraire fixe.

2. Par extension: activité d'un armateur qui place systématiquement son navire sur le marché de l'affrètement.

Anglais: tramping.

ventilation

Voir: Répartition.

\section{Annexe II \\ Liste des termes d' usage recommandé}

Agétac, n.m.

Domaine: Commerce international.

Définition: Abréviation d'Accord général sur les tarifs douaniers et le commerce.

Notes: 1. Cet accord a été conclu en 1947 par 23 pays occidentaux, en vue de réduire les tarifs douaniers, ainsi que les autres entraves aux échanges et d'éliminer les discriminations en matière de commerce international par le jeu de la clause de la nation la plus favorisée. Le nombre actuel des parties contractantes est de 90 .

2. Par extension, ce sigle désigne également l'organisation de fait, née de cet accord, qui siège à Genève et qui a pour mission de veiller au respect des droits et obligations des pays signataires, de régler les différents commerciaux entre ces pays et d'organiser périodiquement des conférences de négociation en vue d'élargir la libéralisation du commerce international.

Anglais : $\quad$ G.A.T.T. (General Agreement on Tariffs and Trade).

bazarette, n.f.

Domaine: Commerce/Réseau de distribution commerciale.

Définition: Petit établissement commercial de moins de $120 \mathrm{~m}^{2}$, dans lequel un personnel réduit offre des produits et services variés de nécessité courante.

Note: Les clients, généralement quotidiens, s'attachent à cette formule qui leur prend peu de temps et concilie une certaine intimité avec les avantages des supermarchés, moyennant un léger surprix.

Anglais: convenience store.

compensation monétaire de groupe, n.f.

Abréviation: CMG, n.f.

Domaine: Finances.

Définition: Technique d'optimisation de la trésorerie d'un groupe, principalement à l'égard du risque de change, et consistant à compenser les dettes et

Anglais : créances, par devises, à l'intérieur du groupe.

évaluation par score, n.f.

Domaine: Finances/Banque.

Synonyme: scorage, n.m. 
Définition: Technique d'évaluation qualitative d'un client emprunteur - particulier ou entreprise - principalement sous l'angle de sa solvabilité.

Anglais: scoring.

scorage

Voir: Évaluation par score.

scorer, $\mathbf{v}$.

Domaine: Banque.

Définition: Évaluer par score.

Anglais: to score.

\section{Annexe III}

Index alphabétique des termes remplacés

Termes de l'annexe

advisory committee

agency fee

back up line

bearish market

brilliant uncirculated

bullet

bullish market

call option

(loss) carry back

cash

cash and carry

cash management

clearing house

committment fee

comfort letter

commercial paper

convenience store

cost and freight (C.F.)

cross default

deficiency payment

deposit

dispatching

drawback

event of default

ex quay ... duty on buyers'

account

ex quay ... duty paid

ex ship

ex works

financial futures

financiers

financial futures market

financiers (MATIF) comité de restructuration I

commission de gestion I

ligne de substitution

marché baissier I

brillant universel (B.U.) I

remboursement in fine I

marché haussier

option d'achat I

report en arrière (de déficit) I

comptant, espèces

payer - prendre I

gestion de trésorerie

chambre de compensation I

commission d'engagement I

lettre de confort I

billet de trésorerie I

bazarette II

coût et fret ( $\mathrm{C}$ et $\mathrm{F})$ I

défaut croisé

paiement différentiel I

dépôt de garantie I

répartition (ou ventilation) I

rembours I

déchéance du terme I

à quai, non dédouané I

à quai, dédouané I

à bord

départ usine

contrats à terme d'instruments I

marché à terme (d'instruments I 
first in, first out (F.I.F.O.) (P.E.P.S.)

fixing

float

forward market

free in and out (F.I.O.)

free on board (F.O.B.)

free on rail (F.O.R.)

free on truck (F.O.T.)

fuel oil

futures market

futures option

GATT (General Agreement on

Tariffs and Trade) AGETAC

gold bullion standard

gold exchange standard

home center

hot money

IBOR (Interbank offered rate)

initial margin (or deposit)

Kennedy round

know how

last in, first out (L.I.F.O.)

(D.E.P.S.)

management fee

marketing

marketing mix

merchandiser

merchandising

multicurrency

netting

new money

off shore

pool

price earning ratio

promissory note

proof

put option

rating

red clause

retail banking

rollover credit

royalty

scoring

small supermarket

splitting

spot credit

spot market premier entré, premier sorti

fixage

flottant (banque et bourse)

marché à terme

bord à bord (B.A.B.)

franco à bord (F.A.B.)

franco wagon

franco camion

fioul

marché de contrats à terme

options sur contrats à terme

Accord général sur les tarifs

et le commerce

étalon-or lingot

étalon de change or

maisonnerie

capitaux flottants

taux interbancaire offert

dépôt de garantie

négociations Kennedy

savoir-faire

dernier entré, premier sorti

commission de chef de file

mercatique

marchéage

marchandiseur

marchandisage

multidevise

compensation monétaire de groupe

crédit de restructuration

extraterritorial

syndicat de prise ferme, tour de table

coefficient de capitalisation

des résultats (C.C.R.)

billet à ordre

épreuve

option de vente

notation

clause rouge

banque de détail

crédit à taux révisable

redevance

évaluation par score, scorage II

supérette

division

crédit ponctuel

marché au comptant

I 
spread

steering committee

stripping

straddle

trade mart

tramping

underwriting fee

underwriting group (or pool)

window-dressing marge

comité de restructuration

démembrement

ordre lié

expomarché

transports maritimes à la demande

commission de garantie, commission

de placement

syndicat de prise ferme

habillage de bilan

Journal Officiel De Le République Française Paris, France 